\title{
The Attitude Tracking Robust Controller for Supercavitating Vehicle Based on Backstepping Method
}

\author{
Chengbin Lian ${ }^{1, a}$, Xueyan Jing $^{2, b}$, Yongjun Hao ${ }^{3, c}$, Xianping Tang ${ }^{4, d}$ \\ ${ }^{1}$ China Ship Research and Development Academy, Beijing, 100101, P.R.China \\ ${ }^{2}$ China Ship Research and Development Academy, Beijing, 100101, P.R.China \\ ${ }^{3}$ China Ship Research and Development Academy, Beijing, 100101, P.R.China \\ ${ }^{4}$ China Ship Research and Development Academy, Beijing, 100101, P.R.China \\ alianchengbin@aliyun.com
}

\begin{abstract}
Keywords: Underwater high-speed vehicle; Adaptive algorithm; Stability control; Variable structure control .

Abstract. The unique characteristics of the supercavitating vehicles is different from the traditional underwater vehicles. For the supercavitating vehicles with nonlinear time-delay dynamics model which containing unmatched uncertainties, the backstepping attitude tracking controller is designed based on backstepping control, adaptive control and sliding mode control. The uncertain factors were estimated by using the adaptive algorithm, and the robustness of the system with uncertainties and external disturbance was improved by using variable structure control. The simulation results show that the system responds rapidly and it has good stability, which shows that the designed controller can be applied to the stability control of underwater high-speed vehicles.
\end{abstract}

\section{Introduction}

The viscous resistance of water is an important factor to limit the speed of underwater vehicle. Supercavitation drag reduction technology capable of forming a parcel sail body cavity which could reduce resistance significantly, and at the same time the underwater vehicle can also has ultra-high speed[1]. Nevertheless, the fluid dynamics of the super-cavitating vehicle is still different from the conventional one. Due to cavitation generation, the pressure center of the vehicle moves forward, and the added mass and damping torque decrease, which make the vehicle body is more sensitive response to outside interference. This reason has brought a lot of technical difficulties to its dynamics modeling, guidance, control and attitude stability[2]. Foreign countries have carried out the research on the problem of dynamic modeling and stability control, and the corresponding results are published. The literature proposes supercavitating vehicle control problems, and gives control based on feedback linearization method[3]. On the basis of the literature [3], the literature [4-6] studies the absolute stability of the longitudinal motion model of the vehicle by means of the bifurcation analysis, and gives the feedback linearization control scheme. Based on the conditions for the existence of sliding force for switch control judgment, the literature [4-6] gives the design scheme of LQR controller. And other literature chose the same as the [7] to determine the basis of the same switch control, and gave a further feedback linearization control scheme for nonlinear model. However, these studies are based on precise model of the supercavitation vehicle, very few of these research focused on the uncertainty model. The uncertainties of the bubble oscillation, fluid dynamic coefficient perturbation, cavitation number perturbation and external disturbance are happened in the actual sailing of the supercavitating vehicle, which are difficult to meet the matching conditions and bright about the control problems of attitude stability.

In this paper, the attitude tracking controller is designed based on Backstepping method, which substitute the continuous sliding mode controller for the traditional sliding mode controller. Mathematical simulation shows that the attitude tracking controller can make the vehicle has good dynamic performance and robustness. 


\section{The Time delay nonlinear mathematical model of underwater high speed vehicle}

Because of the complexity of the high-speed underwater cruising vehicle, this paper mainly studies the longitudinal motion. The vehicle is mainly affected by the lift $F_{c a v}$ which acting on the position of the head cavitator, the gravity $F_{g}$ which acting on center of mass, the lift $F_{f}$ which acting on the rudder and the sliding force $F_{\text {plan }}$ which generated by tail slap between vehicle body and the cavity. Because of the influence of the shape memory effect of the sliding force, the mathematical model of the underwater high speed vehicle has a strong nonlinear and time delay. The expression of the nonlinear sliding force is presented as:

$$
\begin{gathered}
F_{p}=-\rho V^{2}\left(\pi R^{2}\right)\left[1-\left(\frac{R_{c}^{t}-R}{h^{\prime} R+R_{c}^{t}-R}\right)^{2}\right] \frac{1+h^{\prime}}{1+2 h^{\prime}} \alpha \\
h^{\prime}=\left\{\begin{array}{c}
\frac{1}{R}\left[z(t)-\theta(t) l+R-z(t-\tau)-R_{c}^{t}\right], \\
\mathrm{z}(t)+\theta(t) l+R>z(t-\tau)-R_{c}^{t} ; \\
0, \text { no contact } \\
\frac{1}{R}\left[z(t-\tau)+R_{c}^{t}-z(t)-\theta(t) l-R\right], \\
\mathrm{z}(t)+\theta(t) l+R<z(t-\tau)-R_{c}^{t} .
\end{array}\right.
\end{gathered}
$$

Where, $z$ represents dive depth of the vehicle, $w$ represents longitudinal velocity, $\theta$ represents pitch angle, $q$ represents pitch rate, $\delta_{c}, \delta_{f}$ as a control input, $V$ represents velocity of vehicle, $R$ represents the radius column of vehicle, $R_{c}$ represents the radius of cavitation at the end of a vehicle.

Based on the precise mathematical model of the longitudinal motion of the vehicle in [4-6] and the analysis of the uncertainty, let system state is $x_{1}=\left[\begin{array}{ll}z & \theta\end{array}\right]^{T}, x_{2}=\left[\begin{array}{ll}w & q\end{array}\right]^{T}$, control input is $u=\left[\begin{array}{ll}\delta_{f} & \delta_{n}\end{array}\right]^{T}$.

The nonlinear equations can be obtained as following:

$$
\left\{\begin{array}{c}
\dot{x}_{1}=A_{1} x_{1}+x_{2}+d(x, t) \\
\dot{x}_{2}=\left(A_{2}(t, \tau)+\Delta A_{2}\right) x_{2}+\left(B_{0}(t, \tau)+\Delta B(t, \tau)\right) u+ \\
C_{g}+D_{p} F_{p}(x, t, \tau)+f(x, t)
\end{array}\right.
$$

Where, $x_{1}=\left[\begin{array}{ll}z & \theta\end{array}\right]^{T}, x_{2}=\left[\begin{array}{ll}w & q\end{array}\right]^{T}, u=\left[\begin{array}{ll}\delta_{\text {fin }} & \delta_{c}\end{array}\right]^{T}$

$$
A_{1}=\left[\begin{array}{cc}
0 & -V \\
0 & 0
\end{array}\right] A_{2}=\left[\begin{array}{cc}
\frac{-C_{1}-C_{2}}{m V} & \frac{C_{1} L_{c}-C_{2} L_{f}+m V^{2}}{m V} \\
\frac{C_{1} L_{c}+C_{2} L_{f}}{J_{y} V} & \frac{C_{1} L_{c}^{2}+C_{2} L_{f}^{2}}{J_{y} V}
\end{array}\right] B_{0}(t, \tau)=\left[\begin{array}{cc}
-\frac{C_{2}}{m} & -\frac{C_{1}}{m} \\
\frac{C_{2} L_{f}}{J_{y}} & \frac{C_{1} L_{c}}{J_{y}}
\end{array}\right] C_{g}=\left[\begin{array}{l}
g \\
0
\end{array}\right], D_{p}=\left[\begin{array}{cc}
\frac{1}{m} & \frac{L_{f}}{J_{y}}
\end{array}\right]^{T} .
$$

The meaning and parameter setting of related symbols refer to [3-6]. The $\Delta A_{2}, \Delta B(t, \tau)$ represent uncertainty of system matrix $A_{2}$ and control matrix $B_{0}(t, \tau)$ respectively, $f(x, t)$ represent external interference of the vehicle, $d(x, t)$ represent un-matched uncertainty of vehicle, such as $d(x, t)=\left[\begin{array}{lll}\chi_{1}\left(z-z_{d}\right) & \chi_{1}\left(\theta-\theta_{d}\right)\end{array}\right]^{T}$ represent sensor error.

Among them, $\chi_{1} 、 \chi_{2}$ are unknown constant, $z_{d} 、 \theta_{d}$ are reference tracking track.

Assume 1: $\|d(x, t)\| \leq \rho_{1} \kappa_{1}$, where $\rho_{1}$ is unknown bounded positive constant. $\kappa_{1}=\kappa_{1}(x)$ is known smooth function.

Assume 2: There is unknown bounded constant $\gamma<1 、 \vartheta_{1}$ and $\vartheta_{2}$. So that $\|\Delta B\| \leq \gamma\left\|B_{0}\right\|,\left\|\Delta A_{22}\right\| \leq \vartheta_{1}$, $\|f(x, t)\| \leq \vartheta_{2}$ establishment. 


\section{The Attitude tracking controller design based on Backstepping method}

Define $x_{1 d}, x_{2 d}$ as the expected output of equation(3), and suppose it is a bounded continuous differentiable function. Introduce following errors coordinates:

$$
\begin{aligned}
& e_{1}=x_{1}-x_{1 d} \\
& e_{1}=x_{1}-x_{1 d}
\end{aligned}
$$

The following tracking error state equation can be obtained:

$$
\begin{aligned}
& \dot{e}_{1}=A_{1} e_{1}+e_{2}+d(x, t)+A_{1} x_{1 d}+x_{2 d}-\dot{x}_{1 d} \\
& \dot{e}_{2}=\left(A_{2}(t, \tau)+\Delta A_{2}\right) e_{2}+\left(B_{0}(t, \tau)+\Delta B(t, \tau)\right) u+C_{g}+D_{p} F_{p}(x, t, \tau)+f(x, t)+\left(A_{2}(t, \tau)+\Delta A_{2}\right) x_{2 d}-\dot{x}_{2 d}
\end{aligned}
$$

Define $\alpha_{1}$ is expected value for $x_{2 d}$, that is virtual controller to be designed. Define:

$$
\begin{aligned}
& z_{1}=e_{1} \\
& z_{2}=e_{2}-\alpha_{1}
\end{aligned}
$$

From nonlinear system (3)、(8)and(9), it can be get:

$$
\begin{aligned}
& \dot{z}_{1}=A_{1} z_{1}+z_{2}+d(x, t)+A_{1} x_{1 d}+x_{2 d}-\dot{x}_{1 d}+\alpha_{1} \\
& \dot{z}_{2}=\left(A_{2}(t, \tau)+\Delta A_{2}\right) x_{2}+\left(B_{0}(t, \tau)+\Delta B(t, \tau)\right) u+C_{g}+D_{p} F_{p}(x, t, \tau)+f(x, t)-\dot{x}_{2 d}-\dot{\alpha}_{1}
\end{aligned}
$$

Controller design steps are as follows: First step:The design of virtual control. Select sliding mode surface as $S_{1}=z_{1}$, design the following continuous differentiable approximate variable structure virtual controller without regard to $z_{2}$ :

$$
\begin{aligned}
& \alpha_{1}=-\left(\eta_{1} z_{1}+A_{1} z_{1}+A_{1} x_{1 d}+x_{2 d}-\dot{x}_{1 d}\right)-\hat{\rho}_{1} \kappa_{1} \tanh \left(\beta(t) z_{1}\right) \\
& \dot{\hat{\rho}}_{1}=\frac{1}{q_{1}} \kappa_{1}\left\|z_{1}\right\|-\mu_{1}(t) \hat{\rho}_{1}
\end{aligned}
$$

Where, $q_{1}>0, r_{1}>0$ are design parameters, $\hat{\rho}_{1}$ is estimate of the uncertainty, $\mu_{1}(t)$ is the negative feedback which is introduced to make the identification results stable.

$$
\tanh \left(\beta(t) z_{1}\right)=\frac{e^{\beta(t) z_{1}}-e^{-\beta(t) z_{1}}}{e^{\beta(t) z_{1}}+e^{-\beta(t) z_{1}}}
$$

This hyperbolic tangent function is continuously differentiable, when $\beta(t) \rightarrow 0, \tanh \left(\beta z_{1}\right) \rightarrow \operatorname{sgn}\left(z_{1}\right)$. Hyperbolic tangent function replacement symbols function which will cause control signals discontinuous and by selecting function $\beta(t)$, which can make the system keep good robustness, and the steady state error can also meet the requirements. At the same time, the smooth control law can be obtained.

Consider the following Lyapunov function:

$$
V_{1}=\frac{1}{2} z_{1}^{T} z_{1}+\frac{1}{2} q_{1}\left(\rho_{1}-\hat{\rho}_{1}\right)^{2}+q_{1} \mu_{1} r_{1}^{-1} \rho_{1}^{2}
$$

Differentiate $V_{1}$ for time along with (10), it can be got:

$$
\dot{V}_{1}=z_{1}^{T} \dot{z}_{1}-q_{1}\left(\rho_{1}-\hat{\rho}_{1}\right) \dot{\hat{\rho}}_{1}+q_{1} \dot{\mu}_{1} r_{1}^{-1} \rho_{1}^{2}
$$

From (12) (14)、(16), it can be got:

$$
\begin{aligned}
& \dot{V}_{1}=z_{1}^{T}\left(z_{2}+d(x, t)-\eta_{1} z_{1}-\hat{\rho}_{1} \kappa_{1} \tanh \left(\beta(t) z_{1}\right)\right)-q_{1}\left(\rho_{1}-\hat{\rho}_{1}\right)\left(\frac{1}{q_{1}} \kappa_{1}\left\|z_{1}\right\|-\mu_{1}(t) \hat{\rho}_{1}\right)-q_{1} \mu_{1}(t) \rho_{1}^{2} \leq \\
& -z_{1}^{T} \eta_{1} z_{1}+z_{1}^{T} z_{2}+q_{1} \mu_{1}(t)\left(\rho_{1}-\hat{\rho}_{1}\right) \hat{\rho}_{1}-q_{1} \mu_{1}(t) \rho_{1}^{2} \leq-z_{1}^{T} \eta_{1} z_{1}-\frac{1}{2} q_{1} \mu_{1}(t)\left(\rho_{1}^{2}+\hat{\rho}_{1}^{2}\right)+z_{1}^{T} z_{2}
\end{aligned}
$$

Second step: The design of actual control.

For subsystem (11):

$$
\dot{z}_{2}=\left(A_{2}(t, \tau)+\Delta A_{2}\right) x_{2}+\left(B_{0}(t, \tau)+\Delta B(t, \tau)\right) u+C_{g}+D_{p} F_{p}(x, t, \tau)+f(\mathrm{x}, \mathrm{t})-\dot{x}_{2 d}-\dot{\alpha}_{1}
$$

Select the sliding mode surface as $S_{2}=z_{2}$. When we does not consider the uncertainty of the system and external disturbance. According to the $\dot{S}_{2}=-\eta_{2} z_{2}$ ( $\eta_{2}$ is positive definite symmetric matrix), can get the following equivalent control: 
$u_{e q}=-B_{0}^{-1}\left(z_{1}+\eta_{2} z_{2}+A_{2} x_{2}+C_{g}+D_{p} F_{p}(x, t, \tau)-\dot{x}_{2 d}\right)$

Assume 3: $\left\|\Delta A_{2} x_{2}+\Delta B(t, \tau) u_{e q}-\dot{\alpha}_{1}\right\| \leq(1-\gamma) \rho_{2} \kappa_{2}$,

where $\rho_{2}$ is unknown bounded positive constant.

$\kappa_{2}=\kappa_{2}(x)$ is known smooth function. Then adopting the following continuous differentiable approximation variable structure controller:

$$
\begin{aligned}
& u=-B_{0}^{-1}\left(z_{1}+\eta_{2} z_{2}+A_{2} x_{2}+C_{g}+D_{p} F_{p}(x, t, \tau)-\dot{x}_{2 d}\right)-B_{0}^{-1} \hat{\rho}_{2} \kappa_{2} \tanh \left(\beta(t) z_{2}\right) \\
& \dot{\hat{\rho}}_{2}=\frac{1}{q_{2}} \kappa_{2}\left\|z_{2}\right\|-\mu_{2}(t) \hat{\rho}_{2} \\
& \dot{\mu}_{2}(t)=-r_{2} \mu_{2}(t)
\end{aligned}
$$

Where, $q_{2}>0, r_{2}>0$ are design parameters; $\hat{\rho}_{2}$ is estimate of uncertainty; $\mu_{2}(t)$ is negative feedback which to stabilize identification results,

$$
\tanh \left(\beta(t) z_{2}\right)=\frac{e^{\beta(t) z_{2}}-e^{-\beta(t) z_{2}}}{e^{\beta(t) z_{2}}+e^{-\beta(t) z_{2}}}
$$

Consider the following Lyapunov function:

$$
V_{2}=V_{1}+\frac{1}{2} z_{2}^{T} z_{2}+\frac{1}{2}(1-\gamma) q_{2}\left(\rho_{2}-\hat{\rho}_{2}\right)^{2}+(1-\gamma) q_{2} \mu_{2} r_{2}^{-1} \rho_{2}^{2}
$$

Differentiate $V_{1}$ for time along with (11), it can be got:

$$
\dot{V}_{2}=\dot{V}_{1}+z_{2}^{T} \dot{z}_{2}-(1-\gamma) q_{2}\left(\rho_{2}-\hat{\rho}_{2}\right) \dot{\hat{\rho}}_{2}+(1-\gamma) q_{2} \dot{\mu}_{2} r_{2}^{-1} \rho_{2}^{2}
$$

From (20) (22)、(24), it can be got:

$$
\begin{aligned}
& \dot{V}_{2}=-z_{1}^{T} \eta_{1} z_{1}-\frac{1}{2} q_{1} \mu_{1}(t)\left(\rho_{1}^{2}+\hat{\rho}_{1}^{2}\right)+z_{1}^{T} z_{2}+z_{1}^{T} \dot{z}_{2}-(1-\gamma)\left[q_{2}\left(\rho_{2}-\hat{\rho}_{2}\right) \dot{\hat{\rho}}_{2}+q_{2} \dot{\mu}_{2} r_{2}^{-1} \rho_{2}^{2}\right] \\
& =-z_{1}^{T} \eta_{1} z_{1}-\frac{1}{2} q_{1} \mu_{1}(t)\left(\rho_{1}^{2}+\hat{\rho}_{1}^{2}\right)+z_{1}^{T} z_{2}-(1-\gamma) q_{2}\left(\rho_{2}-\hat{\rho}_{2}\right)\left(\frac{1}{q_{2}} \kappa_{2}\left\|z_{2}\right\|-\mu_{2}(t) \hat{\rho}_{2}\right)+ \\
& z_{2}^{T}\left(\begin{array}{l}
\Delta A_{2} x_{2}+\Delta B(t, \tau) u_{e q}-\dot{\alpha}_{1}-z_{1}-\eta_{2} z_{2} \\
-(B+\Delta B) B_{0}^{-1} \hat{\rho}_{2} k_{2} \tanh \left(\beta(t) z_{2}\right)
\end{array}\right)-(1-\gamma) q_{2} \mu_{2} \rho_{2}^{2} \\
& \leq-z_{1}^{T} \eta_{1} z_{1}-z_{2}^{T} \eta_{2} z_{2}-\frac{1}{2} q_{1} \mu_{1}(t)\left(\rho_{1}^{2}+\hat{\rho}_{1}^{2}\right)-\frac{(1-\gamma)}{2} q_{2} \mu_{2}(t)\left(\rho_{2}^{2}-\hat{\rho}_{2}^{2}\right) \leq 0
\end{aligned}
$$

So as to realize the stabilization of the system, it will be asymptotically stable finally.

\section{Simulation analysis}

The model of the longitudinal motion of the supercavitation vehicle described in (3) is studied by the structure parameters of the vehicle as follow:

Gravity acceleration $g=9.81 \mathrm{~m} / \mathrm{s}^{2}$, Vehicle density $\rho_{0}=2000 \mathrm{~kg} / \mathrm{m}^{3}$, Tail rudder similarity coefficient $n=0.5$, Radius of cavitation $R_{n}=0.0191 \mathrm{~m}$, Radius column of vehicle $R=0.0508 \mathrm{~m}$, Total length of vehicle $L=1.8 \mathrm{~m}$, Speed of vehicle $V=75 \mathrm{~m} / \mathrm{s}$, Cavitation number $\sigma=0.03$, body Mass of vehicle $m=22 \mathrm{~kg}$, Moment of inertia $J_{y}=5.1847 \mathrm{kgm}^{2}$.

In order to investigate the effect of the controller under the condition of hydrodynamic coefficient greatly perturbed and large external disturbance, we pull side the hydrodynamic coefficients system $50 \%$, cavitation number perturbed ranges $\pm 20 \%$, and define the interference force and moment are $1 \times \sin (2 \pi t)$, which the amplitude is roughly equivalent to gravity's $10 \%$. We pull side the sliding force $10 \%$, The amplitude of the cavitation bubbles is $0.02\left(R_{c}-R\right), d(x, t)=\left[\chi_{1}\left(z-z_{d}\right) \quad \chi_{1}\left(\theta-\theta_{d}\right)\right]^{T}$, the expectations of $\chi_{1}, \chi_{2}$ are 0.01 . The system initial state are $x(0)=\left[\begin{array}{llll}1 & 0.05 & 0 & 0\end{array}\right]^{T}$.

Taking into account the practical effectiveness of the vehicle, the control law must have the following indicators: the quick response and the limiter feature of control .Since this article mainly study the attitude 
tracking performance of vehicle, the rudder deflection angle and cavitation deflection angle range are taken as $\pm 20^{\circ}$.

In this paper, the attitude tracking controller are designed, which under condition of the adaptive law and the attitude of the initial value of supercavitation vehicle and ignore the noise measuring element. Simulation analysis of attitude tracking for the following reference trajectory as followed:

$x_{1 d}=\left[\begin{array}{ll}z_{d} & \theta_{d}\end{array}\right]^{T}, x_{2 d}=\left[\begin{array}{ll}w_{d} & q_{d}\end{array}\right]^{T}$. Where, $z_{d}=\sin (t), \theta_{d}=-\frac{\dot{z}_{d}}{V}, w_{d}=0, q_{d}=\dot{\theta}_{d}$.

After the numerical simulation of above control method, the state of the system response curve, a control law curve and the tail rudder wet rate curve are shown in Figure 1, which point line and solid line represents reference trajectory and the system response curve respectively. From the simulation results, we can see that the Backstepping method is used to design the supercavitation vehicle attitude tracking controller, the vehicle complete the attitude tracking task so fast, and error within the allowable range. From the point of control precision and stability, the inhibition of mismatched uncertainty and external disturbance can satisfy the requirements of the system design at the same time.
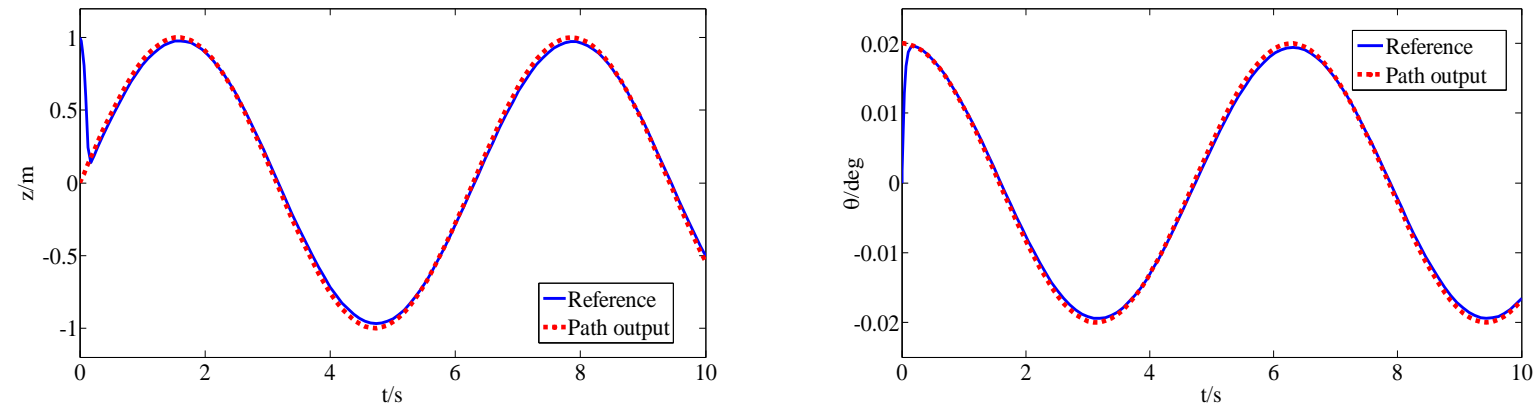

(a) The response of the state
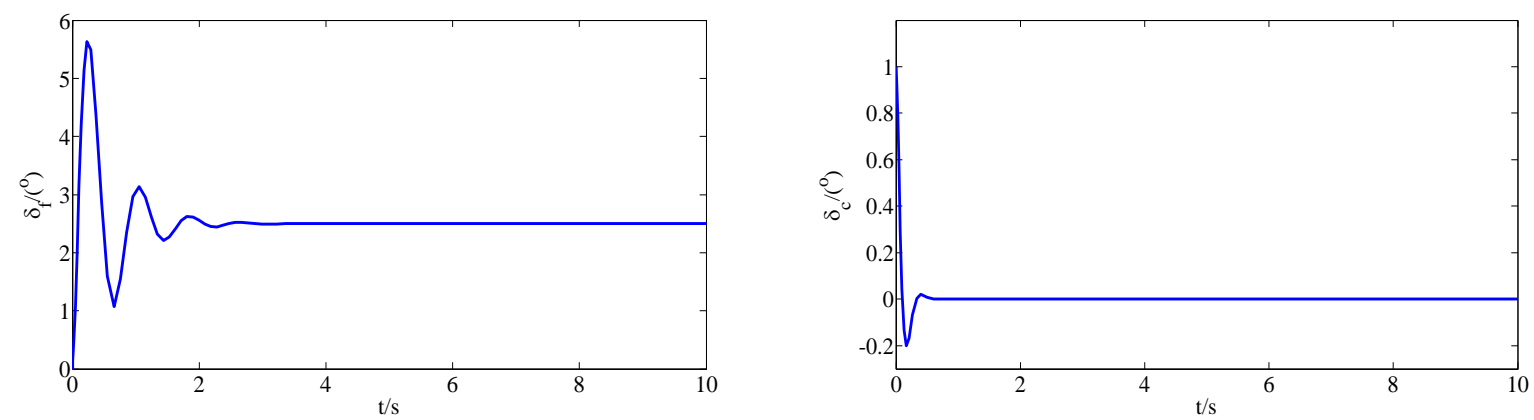

(b) Time history of the control

Figure 1. Attitude maneuver curve

\section{Conclusions}

This paper designed the attitude tracking controller based on Backstepping method for the longitudinal motion equation of the vehicle with non matching uncertainties. The whole design process is divided into two steps: the first step is to design a virtual controller use variable structure control technology. In order to reduce the conservatism of the controller, the adaptive control law is given. The second step is to design a robust attitude tracking controller based on Backstepping method and variable structure control technology. Finally, the proposed control method is used to simulate the model of the supercavitation vehicle. Simulation results further verify the correctness of the proposed design method and theoretical analysis, and have a certain engineering application prospect. 


\section{Acknowledgements}

This study has been supported by the Innovation Fund of China Ship Research and Development Academy.

\section{References}

[1] Savchenko Y.:Supercavitation problems and perspectives Fourth International Symposium on Cavitation, California: California Institute of Technology. Pasadena, CA USA, No. 003,( 2001).

[2] Kirschner I, Uhlman J.: Overview of high-speed supercavitating vehicle control. AIAA Guidance, Navigation, and ControlConference and Exhibit. Keystone, Colorado: American Institute of Aeronautics and Astronautics Inc, p:3100-3116,(2006)..

[3] Dzielski J, Kurdila A. A benchmark: Journal of Vibration Control, vol.9,(2003). P.791-804

[4] Lin G J, Balachandran B, Eyad H A. Nonlinear dynamics and control of supercavitating bodies, AIAA Guidance, Navigation, and Control Conference 2006, Keystone, Colorado.p: 3151-3164,(2006).

[5] Lin G J, Balachandran B, Eyad H A. IEEE J Oceanic, vol.32(4): (2007) ,p:753-761

[6] Lin G J, Balachandran. Eyad H A. Journal Dynamic System and Measure Control, vol.130(2), (2008), article .No. 021003.

[7] Shao Y F, Mesbahi M, Balas G. "Planing, switching and supercavitating flight control". AIAA Guidance, Navigation, and Control Conference. Reston, VA, USA: AIAA, 2003, article No. 5724. 